\title{
Prognostic Value of Extranodal Tumor Extension in Papillary Thyroid Carcinoma: Proposal for Upstaging of Cases with Extranodal Tumor Extension
}

\author{
Yasuhiro Ito $^{1} \cdot$ Akira Miyauchi $^{1} \cdot$ Hiroo Masuoka $^{1} \cdot$ Takuya Higashiyama $^{1} \cdot$ \\ Minoru Kihara $^{1} \cdot$ Akihiro Miya $^{1}$
}

Published online: 11 October 2019

(C) The Author(s) 2019

\begin{abstract}
Background Recently, we have created a revised version of the eighth edition of the tumor-node-metastasis classification for papillary thyroid carcinomas (PTCs) by subdividing the T4a (T4a1 [moderate] and T4a2 [significant]) and $N(\mathrm{~N} 1[N \leq 3 \mathrm{~cm}]$ and $\mathrm{N} 2[N>3 \mathrm{~cm}])$ classifications. This re-staging better stratified patient outcomes. In this study, we investigated the prognostic significance of extranodal tumor extension (LNEx) in PTC.

Methods Five thousand six hundred and eighty-three patients with PTC surgically treated in Kuma Hospital were enrolled. We evaluated LNEx based on intraoperative findings.

Results One hundred and twenty-seven patients (2\%) displayed LNEx. In contrast to what we observed for extrathyroid extension, the prognostic value of LNEx did not change based on the organ that had been invaded, and we therefore analyzed LNEx patients as a single group. In patients aged 55 or older, LNEx independently affected patients' prognoses, as did T4a2 and N2. The cancer-specific survival (CSS) of patients in Stage I but having LNEx demonstrated the similar prognosis to patients in Stage II. Further, in the subset analysis for Stage II patients aged 55 or older, LNEx had a significant prognostic value for CSS in both the univariate and multivariate analyses, as did N2. The CSS of Stage II patients aged 55 or older with LNEx did not differ from that of Stage III patients.

Conclusions It is appropriate that, similar to T4a2 or N2 patients, LNEx-positive patients younger than 55 years in Stage I and those aged 55 or older in Stage II are re-staged to II and III, respectively.
\end{abstract}

\section{Introduction}

The eighth edition of tumor-node-metastasis (TNM) classification was published in 2017 [1, 2], which includes several important changes, such as the age cutoff being set at 55 years instead of 45 years, T1-3N1M0 patients $\geq 55$ years being downstaged from Stage III or IV to Stage II, and patients with extrathyroid extension corresponding to T4a being downstaged from Stage IVA to Stage III. Previous studies, including ours, showed that the eighth

Yasuhiro Ito ito01@kuma-h.or.jp

1 Department of Surgery, Kuma Hospital, 8-2-35, Shimoyamate-dori, Chuo-ku, Kobe 650-0011, Japan edition more keenly reflected the patients' outcomes with differentiated thyroid carcinoma than the seventh edition [3-6].

Recently, we have proposed a revised TNM staging system (re-Stage) for papillary thyroid carcinoma (PTC). We divided T4a and N1 into two categories based on the organs to which the PTC invades and the size of metastasis, respectively, and showed that the re-Stage reflected patients' prognoses more keenly than the eighth edition [7]. However, tumor extension to adjacent organs can originate not only from primary lesions but also from metastatic nodes, although this is rare. Indeed, previous studies, including some from our institution, showed the prognostic significance of extranodal tumor extension (LNEx) in PTC [8-13]. In this study, therefore, we investigated the 
prognostic significance of LNEx and the possibility of upstaging of LNEx-positive cases using a large series of PTC patients.

\section{Materials and methods}

\section{Patients}

Five thousand six hundred and eighty-three patients with PTC who underwent surgery at the Kuma Hospital from 1988 to 2005 were enrolled (follow-up periods 6-345 months, median 175 months). We excluded patients with other thyroid malignancies. No Stage IVA patients were enrolled in our series because $\mathrm{T} 4 \mathrm{~b}$ cases are very rare. We obtained informed consent to participate from all patients in advance of the postoperative follow-up; all patients agreed to be followed up by questionnaire even after leaving the hospital. Since this study is retrospective, we did not obtain approval from the ethical committee, subject to the decision of our ethics board. This series is the same as that in our previous study regarding the revised TNM eighth edition [7], but based on further investigation of patients' prognoses, the number of patients who died of thyroid carcinoma increased from 110 to 116 , as described below (postoperative follow-up).

\section{Categorization of LNEx}

We regarded PTC invasion from metastatic nodes to adjacent organs corresponding to $\mathrm{T} 4 \mathrm{a}$ as positive for $\mathrm{LNEx}$ based exclusively on intraoperative findings. PTC invasion from metastatic nodes only to surrounding fat tissue was not categorized as LNEx positivity. None of the patients showed extranodal tumor extension to organs corresponding to $\mathrm{T} 4 \mathrm{~b}$.

We classified LNEx into two grades: LNEx1, extension to the tracheal adventitia and cartilage, esophageal muscle layer, recurrent laryngeal nerve, cricothyroid muscle, and inferior constrictor muscle, and LNEx2, extension to other organs such as thyroid cartilage, the subcutaneous soft tissues, larynx, tracheal mucosa, esophageal mucosa, jugular vein, brachiocephalic veins, and sternocleidomastoid muscle, which was the same manner as the subdividing of T4a [7].

\section{Surgery}

Total thyroidectomy, subtotal thyroidectomy, isthmectomy, and hemithyroidectomy were performed for 2970 , 480,50 , and 2183 patients, respectively. Prophylactic or therapeutic central node dissection was also performed for 5418 patients. Further, 4280 patients underwent uni- or bilateral modified radical neck dissection and 13 underwent therapeutic dissection of upper mediastinal compartment.

\section{Radioactive iodine therapy}

Radioactive iodine (RAI) therapy was performed for 47 of 66 patients with clinical distant metastases. RAI therapy was not performed for the remaining 19 because of poor performance status of patients and their refusal. Seventynine patients with M0 underwent administration of RAI $\geq 50 \mathrm{mCi}$ for adjuvant therapy or remnant thyroid ablation after total thyroidectomy.

\section{Follow-up of patients after surgery}

All patients visited our hospital for blood examination and imaging studies, including ultrasonography, at least once per year. Chest radiography, computed tomography, and bone scintigraphy were also used for follow-up at the physicians' discretion. To date, 377 patients have died of various causes, including 116 of thyroid carcinoma. The incidence of thyroid carcinoma death significantly differed according to patients' age at the initial surgery: Only 26 of 3433 patients $<55$ years $(0.8 \%)$ died of thyroid carcinoma, whereas 90 of 2250 patients $(4.9 \%) \geq 55$ years did.

\section{Statistical analyses}

For univariate analysis, the Kaplan-Meier method and logrank test were adopted. The Cox proportional hazard model was used for multivariate analysis. Both were performed by the StatView (SAS, Tokyo, Japan). We regarded $p$-values smaller than 0.05 as statistically significant.

\section{Results}

\section{Background and clinicopathological features of the present series}

Table 1 shows the backgrounds and clinicopathological features of the 5683 patients enrolled in this study. Of these, 127 patients (2\%) were classified as LNEx-positive based on intraoperative gross findings, and at least partial resection of the organs that metastatic nodes invaded was required for curative resection. The incidence of LNEx was $2 \%$, much lower than that of extrathyroid extension from the primary lesion (14\%). Metastatic nodes had invaded the recurrent laryngeal nerve in 36 patients, internal jugular vein in 89 patients, external jugular vein in 2 patients, vagus nerve in 4 patients, accessory nerve in 7 patients, phrenic nerve in 5 patients, sternomastoid muscle in 2 patients, sympathetic truck in 4 patients, supraclavicular 
Table 1 Backgrounds and clinicopathological features of the 5683 patients

\begin{tabular}{|c|c|c|}
\hline & Characteristic & No. of patients $(\%)$ \\
\hline \multirow[t]{2}{*}{ Age } & $\geq 55$ years & $2250(40 \%)$ \\
\hline & $<55$ years & $3433(60 \%)$ \\
\hline \multirow[t]{2}{*}{ Gender } & Male & $659(11 \%)$ \\
\hline & Female & $5024(89 \%)$ \\
\hline \multirow[t]{5}{*}{ AJCC stage ${ }^{a}$} & I & $4846(85 \%)$ \\
\hline & II & $403(7.2 \%)$ \\
\hline & III & $406(7.2 \%)$ \\
\hline & IVA & 0 \\
\hline & IVB & $28(0.5 \%)$ \\
\hline \multirow[t]{5}{*}{ Re-Stage $^{\mathrm{b}}$} & I & $4721(83 \%)$ \\
\hline & II & $798(14 \%)$ \\
\hline & III & $136(2.5 \%)$ \\
\hline & IVA & 0 \\
\hline & IVB & $28(0.5 \%)$ \\
\hline \multirow[t]{2}{*}{$M$} & M1 & $66(1 \%)$ \\
\hline & M0 & $5617(99 \%)$ \\
\hline \multirow[t]{4}{*}{ Extrathyroid extension } & $\mathrm{T} 4 \mathrm{a} 2$ & $112(2 \%)$ \\
\hline & T4a1 & $595(11 \%)$ \\
\hline & $\mathrm{T} 3 \mathrm{~b}$ & $40(1 \%)$ \\
\hline & Negative & $4936(86 \%)$ \\
\hline \multirow[t]{3}{*}{ Extranodal tumor extension (LNEx) } & $\mathrm{LNEx} 2^{\mathrm{c}}$ & $33(0.6 \%)$ \\
\hline & $\operatorname{LNEx} 1^{\mathrm{d}}$ & $94(1.4 \%)$ \\
\hline & Negative & $5556(98 \%)$ \\
\hline \multirow[t]{2}{*}{ Tumor size } & $>4 \mathrm{~cm}$ & $558(10 \%)$ \\
\hline & $\leq 4 \mathrm{~cm}$ & $5125(90 \%)$ \\
\hline \multirow[t]{3}{*}{$N$} & $\mathrm{~N} 2$ & $192(3 \%)$ \\
\hline & N1 & $1025(18 \%)$ \\
\hline & No & $4567(79 \%)$ \\
\hline
\end{tabular}

${ }^{a}$ American Joint Committee on Cancer eighth edition, ${ }^{b}$ revised stage proposed in our previous study [7], ${ }^{\mathrm{c}}$ corresponding to T4a2, ${ }^{\mathrm{d}}$ corresponding to $\mathrm{T} 4 \mathrm{a} 1$

nerve in 4 patients, brachiocephalic vein in 1 patient, subclavian vein in 1 patient, and submandibular gland in 1 patient. Seventeen patients showed LNEx to two or more organs. Based on the subclassification of extrathyroid extension in the previous study [7], we divided LNEx into two categories, LNEx1 and LNEx2, having extranodal tumor extension to other organs corresponding to T4a1 and $\mathrm{T} 4 \mathrm{a} 2$, respectively. Ninety-four patients were classified as LNEx1, and 33 were classified as LNEx2.

\section{Lack of difference in prognosis between LNEx1 and LNEx2 patients}

We divided LNEx into two categories, LNEx1 and LNEx2, as described in the patients and methods, and compared the differences in CSS between LNEx1 and LNEx2 patients in patients younger than 55 years and those at 55 years or older. The CSS of LNEx2 patients was similar to that of LNEx1 patients in either subset $(p=0.2493$ and $p=0.6074$, respectively). Therefore, we assessed LNEx patients as a single group without subclassification for further analyses.

\section{The relationship between $\mathrm{N}$ and LNEx}

Fifty (26\%) of 192 N2 patients were LNEx-positive, as were $67(7 \%)$ of 1025 N1 patients. Ten of 4466 N0 patients $(0.2 \%)$ were also diagnosed as LNEx-positive based on intraoperative findings. Therefore, the incidence of LNEx was significantly related to $N$ status $(p<0.0001)$. 


\section{Subset analysis of patients younger than 55 years}

On univariate analysis for patients younger than 55 years, LNEx positivity $(N=72 ; p<0.0001$, Fig. 1a), M1 $(N=38 ; p<0.0001), \quad \mathrm{N} 2 \quad(N=114 ; p<0.0001), \mathrm{N} 1$ $(N=651 ; p=0.0161), \mathrm{T} 4 \mathrm{a} 2(N=28 ; p<0.0001), \mathrm{T} 4 \mathrm{a} 1$ $(N=252 ; \quad p=0.0021), \quad$ and $\quad T>4 \mathrm{~cm} \quad(N=334$; $p=0.0006)$ significantly affected CSS. However, no T3b patients died of PTC, indicating that T3b was not related to prognosis in patients younger than 55 years. Next, we performed a multivariate analysis for factors having a prognostic significance on univariate analysis. Not only M1 $(p=0.0001$, odds ratio $[\mathrm{OR}]=8.130)$, but also $\mathrm{T} 4 \mathrm{a} 2$ $(p=0.0003, \quad \mathrm{OR}=10.638), \quad \mathrm{N} 2 \quad(p<0.0001, \quad \mathrm{OR}=$ $12.821), \quad \mathrm{N} 1 \quad(p=0.0417, \quad \mathrm{OR}=3.155)$, and LNEx $(p=0.0326$, OR $=12.195)$ had independent prognostic value for CSS (Table 2). As we showed in our previous study, the CSS of Stage I patients with T4a2 or N2 did not

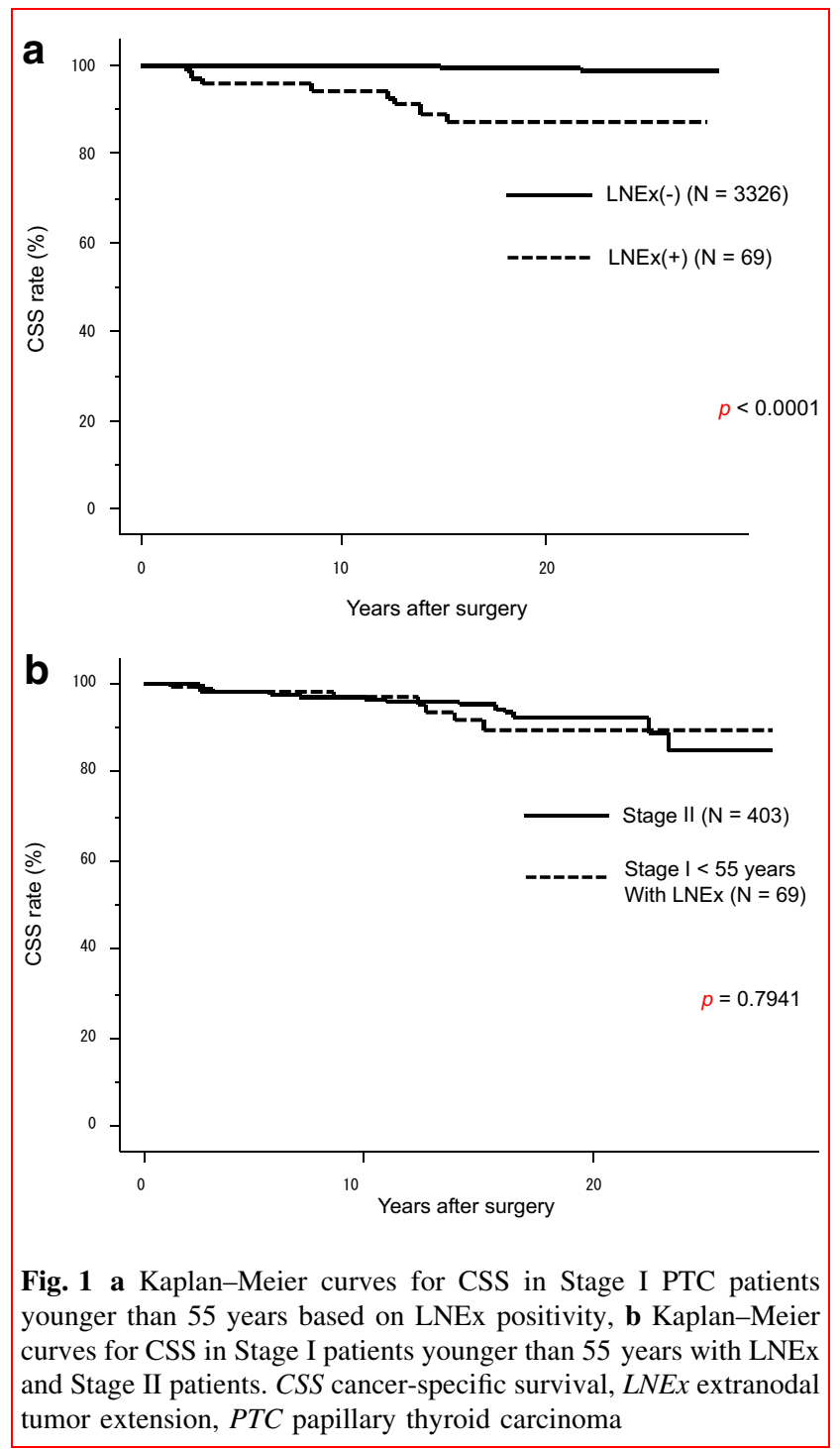

Table 2 Multivariate analysis for factors affecting CSS in PTC patients $<55$ years

\begin{tabular}{lcc}
\hline Variables & $p$ value & Odds ratio $(95 \% \mathrm{CI})$ \\
\hline $\mathrm{M} 1$ & 0.0001 & $8.130(2.809-23.810)$ \\
$\mathrm{T} 4 \mathrm{a} 1$ & 0.5420 & $1.391(0.481-1.391)$ \\
$\mathrm{T} 4 \mathrm{a} 2$ & 0.0003 & $10.638(2.950-38.462)$ \\
$\mathrm{N} 1$ & 0.0417 & $3.155(1.044-9.524)$ \\
$\mathrm{N} 2$ & $<0.0001$ & $12.821(4.098-40.000)$ \\
$T>4 \mathrm{~cm}$ & 0.0585 & $2.283(0.971-5.763)$ \\
LNEx & 0.0326 & $12.195(4.310-34.483)$ \\
\hline
\end{tabular}

CSS cancer-specific survival, PTC papillary thyroid carcinoma, CI confidence interval, $L N E x$ extranodal tumor extension

differ from CSS of Stage II patients [7], and we proposed that patients having these features are upstaged to Stage II in the re-staging system. As shown in Fig. 1b, the CSS of Stage I LNEx-positive patients younger than 55 years did not significantly differ from that of Stage II patients $(p=0.7941)$, indicating that these patients are also strong candidates for upstaging to re-Stage II.

\section{Subset analysis of Stage II patients aged 55 years or older}

On univariate analysis for Stage II patients $\geq 55$ years $(N=365)$, patients with LNEx $(N=25 ; p=0.0016)$ and those with $\mathrm{N} 2(N=37 ; p=0.0013)$ had significantly poorer CSS than patients without these features (Fig. 2a). However, patients with T3b $(N=24 ; p=0.0595)$, $T>4 \mathrm{~cm} \quad(N=106 ; p=0.9543)$, and $\mathrm{N} 1 \quad(N=236$; $p=0.1887)$ did not have worse CSS. On multivariate analysis, both LNEx $(p=0.0456, \mathrm{OR}=3.160)$ and N2 $(p=0.0298, \mathrm{OR}=3.546)$ were independent prognosticators (Table 3). The CSS of N2 patients with Stage II was similar to that of Stage III patients [7]. Similarly, the CSS of patients in Stage II with LNEx and those in Stage III did not significantly differ ( $p=0.4431$; Fig. $2 b)$. Therefore, Stage II patients with LNEx should be upstaged to Stage III in the re-staging system.

\section{Discussion}

In this study, we demonstrated that (1) LNEx was a relatively rare event, occurring in only $2 \%$ of PTC patients, (2) the prognosis of patients with LNEx did not significantly vary according to which organs the metastatic nodes had invaded, (3) in patients younger than 55 years, LNEx independently affected CSS, and the CSS of LNEx-positive patients did not differ from that of Stage II patients, and (4) 


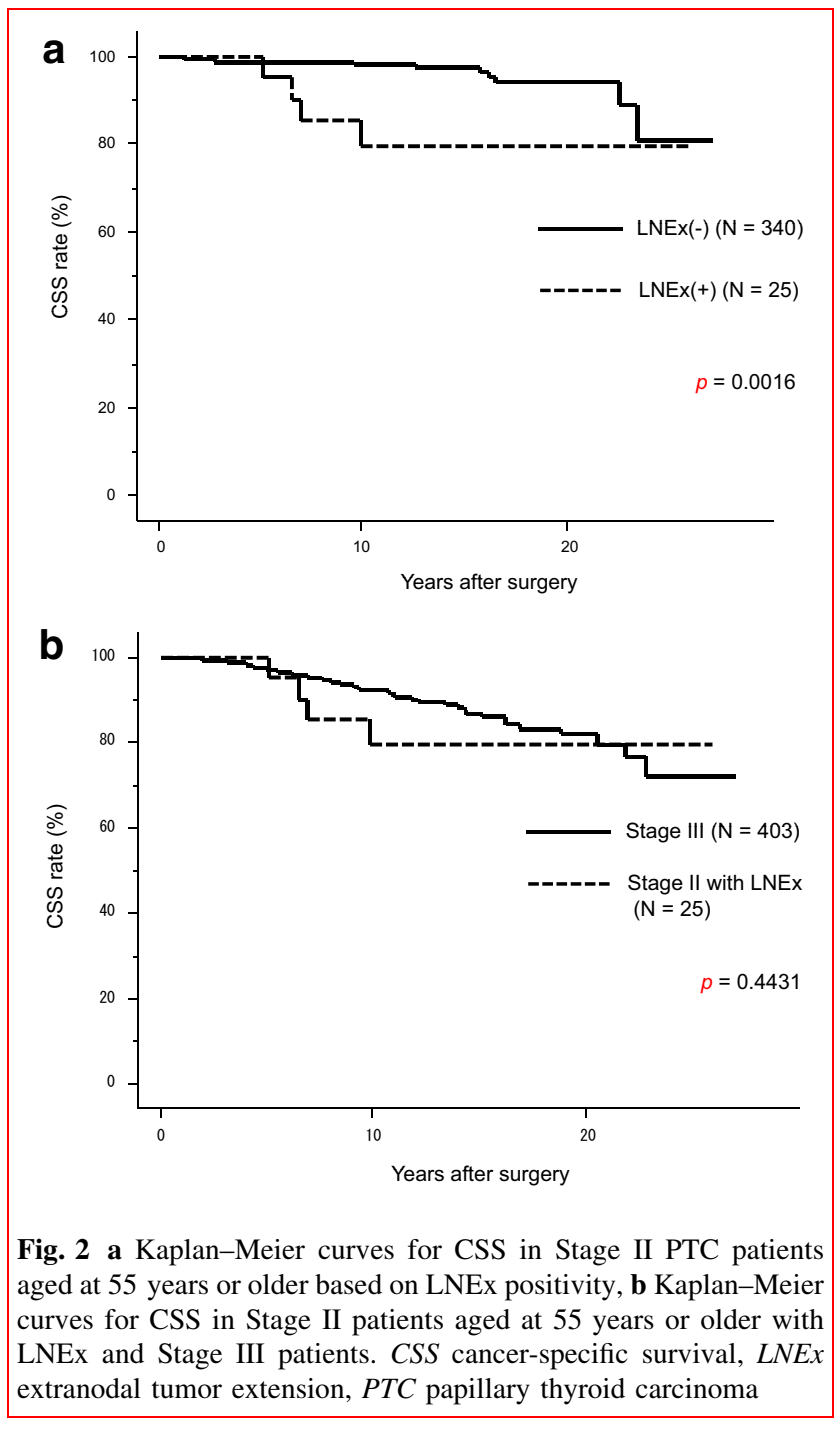

in Stage II patients aged at 55 years or older, LNEx-posi-

Table 3 Multivariate analysis for factors affecting CSS in Stage II PTC patients $\geq 55$ years

\begin{tabular}{lll}
\hline Variables & $p$-value & Odds ratio $(95 \% \mathrm{CI})$ \\
\hline LNEx & 0.0455 & $3.160(1.025-11.628)$ \\
N2 & 0.0298 & $3.546(1.133-11.111)$ \\
\hline
\end{tabular}

CSS cancer-specific survival, $P T C$ papillary thyroid carcinoma, $C I$ confidence interval, LNEx extranodal tumor extension

tive patients had significantly poorer CSS than LNExnegative patients and had similar CSS to Stage III patients.

Previous studies have shown the prognostic significance of LNEx from both clinical and pathological aspects [8-13], and the American Thyroid Association guidelines adopted pathological LNEx as a high-risk feature of differentiated thyroid carcinoma [14]. However, LNEx has not yet been adopted in the TNM classification. This may be because LNEx is not a common event and its incidence is much lower than that of extrathyroid extension from primary lesions.

In contrast to $\mathrm{T} 4 \mathrm{a}$, we could not establish any significant difference in CSS between LNEx1 and LNEx2 patients aged $<55$ years $(p=0.2493)$ or those aged $\geq 55$ years $(p=0.6074)$. Why this discrepancy occurs remains to be elucidated, but a significant volume of lymph node metastasis is needed for this phenomenon, indicating that LNEx reflects two aggressive characteristics of PTC: significant invasive character and extensive lymph node metastasis. This might explain, at least in part, why LNExpositive tumors generally have very highly aggressive features. We analyzed LNEx-positive patients as a single group without subclassification and found that LNEx had a very strong impact on CSS, similar to other factors, such as T4a2 and N2. Stage I patients with LNEx and M0 showed a similar CSS to Stage II patients, and the CSS of Stage II patients positive for LNEx did not differ from that of Stage III patients. These results suggest that patients with LNEx are strong candidates for upstaging.

In multivariate analysis of patients $<55$ years, the ORs of LNEx, N2, and T4a2 were even higher than the OR of M1. M1 is a prominent prognostic factor for CSS, regardless of patient age. However, the CSS of PTC patients $<55$ years is generally good. In our series, only 26 of 3433 patients $<55$ years $(0.8 \%)$ died of thyroid carcinoma, compared to 90 of 2250 patients $\geq 55$ years (4.9\%). The small number of patients $<55$ years who died of thyroid carcinoma may cause some bias. Alternatively, RAI therapy is known to be effective especially for young M1 patients, indicating that the prognostic value of M1 in patients $<55$ years is lower than that in patients $\geq 55$ years, and in these patients, M1 has a prognostic value similar to that of other factors, such as LNEx, T4a2, and N2.

Our study has some limitations. First, this is a retrospective study. Second, LNEx is relatively rare, and its incidence was lower than that of extrathyroid extension. Therefore, analyses including more patients are needed to more accurately elucidate how LNEx affects prognosis. If analyzed using a large number of cases, the prognoses of PTC patients may differ according to which organs metastatic nodes extend. In this study, the classification of LNEx1 and LNEx2 used the same criteria as that for T4a1 and $\mathrm{T} 4 \mathrm{a} 2$, but extranodal tumor extension can occur to organs that are not likely to be invaded by primary lesions, such as the submandibular gland. Here, we classified such cases as LNEx2, but future studies should review whether this is the correct classification. Last, the therapeutic strategies administered to patients enrolled in this study significantly differ from those currently administered. In 
our series, the number of patients who received RAI therapy or ablation was small $(N=79)$.

In summary, in all patients ( $<55$ years and $\geq 55$ years), LNEx showed a significant prognostic value for CSS. Patients with LNEx may be candidates for upstaging. However, further investigation with a larger number of patients having LNEx is required for more detailed analysis, such as the difference in prognoses of LNEx-positive patients according to which organs metastatic lymph nodes extend.

Open Access This article is distributed under the terms of the Creative Commons Attribution 4.0 International License (http://crea tivecommons.org/licenses/by/4.0/), which permits unrestricted use, distribution, and reproduction in any medium, provided you give appropriate credit to the original author(s) and the source, provide a link to the Creative Commons license, and indicate if changes were made.

\section{References}

1. Amin MB, Edge S, Greene F et al (eds) (2017) AJCC cancer staging manual (8th). Springer, New York

2. Edge S, Byrd DR, Compton CC et al (eds) (2010) AJCC cancer staging manual (7th). Springer, New York

3. Ito Y, Miyauchi A, Hirokawa M et al (2018) Prognostic value of the 8th edition of the tumor-node-metastasis classification for patients with papillary thyroid carcinoma: a single-institution study at a high-volume center in Japan. Endocr J 65:707-718

4. Nam SH, Bae MR, Roh JL et al (2018) A comparison of the 7th and 8th editions of the AJCC staging system in terms of predicting recurrence and survival in patients with papillary thyroid carcinoma. Oral Oncol 87:158-164

5. Nava CF, Zanella AB, Scheffei RS et al (2019) Impact of the updated TNM staging criteria on prediction of persistent disease in a differentiated thyroid carcinoma cohort. Arch Endocrinol Metab 63:5-11

6. Ito Y, Hirokawa M, Jikuzono T et al (2007) Extranodal tumor extension to adjacent organs predicts a worse cause-specific survival in patients with papillary thyroid carcinoma. World $\mathbf{J}$ Surg 31:1194-1201. https://doi.org/10.1007/s00268-007-9042-2

7. Ito Y, Miyauchi A, Kihara M et al (2019) Subclassification of tumor extension and nodal metastasis in papillary thyroid cancer to improve prognostic accuracy of the eighth edition of the tumor-node-metastasis classification. World J Surg. https://doi. org/10.1007/s00268-019-05120-w (Epub ahead of print)

8. Ito Y, Ichihara K, Masuoka $\mathrm{H}$ et al (2010) Establishment of an intraoperative staging system (iStage) by improving UICC TNM classification system for papillary thyroid carcinoma. World $\mathrm{J}$ Surg 34:2570-2580. https://doi.org/10.1007/s00268-010-0710-2

9. Alpert EH, Wenig BM, Dewey EH et al (2015) Size distribution of metastatic lymph nodes with extranodal extension in patients with papillary thyroid cancer: a pilot study. Thyroid 25:238-241

10. Wu MH, Shen WT, Gosnell J et al (2015) Prognostic significance of extranodal extension of regional lymph node metastasis in papillary thyroid cancer. Head Neck 37:1336-1343

11. Suh S, Pak K, Seok JW et al (2016) Prognostic value of extranodal extension in thyroid cancer: a meta-analysis. Yonsei Med J 57:1324-1328

12. Kim JW, Roh JL, Gong G et al (2017) Extent of extrathyroidal extension as a significant predictor of nodal metastasis and extranodal extension in patients with papillary thyroid carcinoma. Ann Surg Oncol 24:460-468

13. Roh JL, Park JW, Jeong J et al (2017) Extranodal extension of lymph node metastasis as a prognostic indicator of recurrence and survival in papillary thyroid carcinoma. J Surg Oncol 116:450-458

14. Haugen BR, Alexander EK, Bible KC et al (2016) 2015 American Thyroid Association management guidelines for adult patients with thyroid nodules and differentiated thyroid cancer: the American Thyroid Association guidelines task force on thyroid nodules and differentiated thyroid cancer. Thyroid 26:1-133

Publisher's Note Springer Nature remains neutral with regard to jurisdictional claims in published maps and institutional affiliations. 